\title{
Structural insights into the TRIM family of ubiquitin E3 ligases
}

Cell Research (2014) 24:762-765. doi:10.1038/cr.2014.46; published online 11 April 2014

\section{Dear Editor,}

TRIM proteins play important roles in a wide range of biological processes, including cell proliferation, differentiation, development, apoptosis, oncogenesis and innate immunity $[1,2]$. The N-terminal regions of all TRIM proteins contain a RING finger domain followed by one or two B-box domains and a coiled-coil domain (CCD). The RING-finger domain comprises conserved cysteine and histidine residues that bind two zinc atoms in a 'cross-brace' arrangement, and is essential for recruiting the ubiquitin-charged ubiquitin-conjugating enzymes (E2 Ub). The B-box domains, differing in both the number and spacing of the conserved cysteine and histidine residues [3], are typically composed of B1 and B2 domains, but some TRIM members only contain a B2 domain. CCD following the B-box domains has been proposed to mediate protein-protein interactions, particularly homomeric and heteromeric interactions by forming intertwining helices among TRIM family and other proteins $[4,5]$.

Unlike some ubiquitin ligases that form multi-subunit complexes and catalyze ubiquitination with the help of other subunits [6], TRIM proteins can exert their ubiquitin ligase activity as single-component ubiqutin ligases $[7,8]$. However, the underlying mechanism remains unknown. To understand how the highly conserved CCDs contribute to TRIM-catalyzed unbiquitination, we determined a crystal structure of the CCD (residues 143 to 321 ) of human TRIM69 at $2.15 \AA$ resolution (Figure 1A, 1B and Supplementary information, Figure S1). The structure reveals that TRIM69 CCD forms a homodimer in an anti-parallel orientation (Figure 1B). Each monomer contains three $\alpha$-helices ( $\alpha 1-\alpha 3)$. The N-terminal 112 residues (152 to 263 ) form the $\alpha 1$ helix with the length of about $168 \AA$. The $\alpha 2$ helix containing residues 266 to 288 folds back to form a 3-helix bundle with the two $\alpha 1$ helices of the dimer (Figure 1B and 1C). The short $\alpha 3$ helix (residues 305 to 318 ) is almost perpendicular to the long axis of the two long $\alpha 1$ helices (Figure $1 \mathrm{C}$ and Supplementary information, Figure S2A). In TRIM69, a conserved PRY/SPRY domain (also known as B30.2 domain) follows the CCD. Structural comparison of homologous PRY/SPRY domains in different species reveals that they share a highly similar fold (backbone rmsd of 0.65-1.25 $\AA$ ), despite their low sequence identity (ranging from $15.7 \%$ to $24.7 \%$ ) (Supplementary information, Figure S2B and S2C). Interestingly, most of them contain a short $\alpha$-helix at their N-termini (Supplementary information, Figure S2B), which appears equivalent to the $\alpha 3$ helix in our CCD structure. Indeed, structural modeling shows that the configuration of $\alpha 3$ allows the PRY/SPRY domain to protrude from the rod-like coiled coil without steric clashing (Figure 1B, 1C and Supplementary information, Figure S2A).

The dimerization interface between the two monomers is mainly hydrophobic (Supplementary information, Figure S3). A set of hydrophobic residues at this interface are highly conserved among TRIM proteins (Figure 1C and Supplementary information, Figure S4), suggesting that homo- or hetero-dimerization is a conserved mechanism among the TRIM proteins. Further supporting this notion, crosslinking studies showed that purified recombinant TRIM proteins formed dimers as well as higher oligomers (Supplementary information, Figure S5).

TRIM25 (Supplementary information, Figure S6A) has well-defined physiological substrates. Both TRIM25 and TRIM69 belong to subfamily IV [1], and their expression is up-regulated by many cytokines [9]. Previous studies have established that TRIM25 ubiquitinates the Caspase Activation and Recruitment Domains (CARDs) of RIG-I [7]. Recombinant full-length (FL) TRIM25 protein was eluted with an apparent molecular weight of about $300 \mathrm{kD}$ on a gel-filtration column (Supplementary information, Figure S6B), which is larger than the predicted dimer. However, static light scattering result showed that dimers existed in solution (Supplementary information, Figure S6C). The larger apparent molecular weight of TRIM25 in gel filtration is likely due to an elongated shape of the protein molecule. The 2D classification of raw negative staining electron microscopy (EM) particles of the FL TRIM25 protein confirmed that 


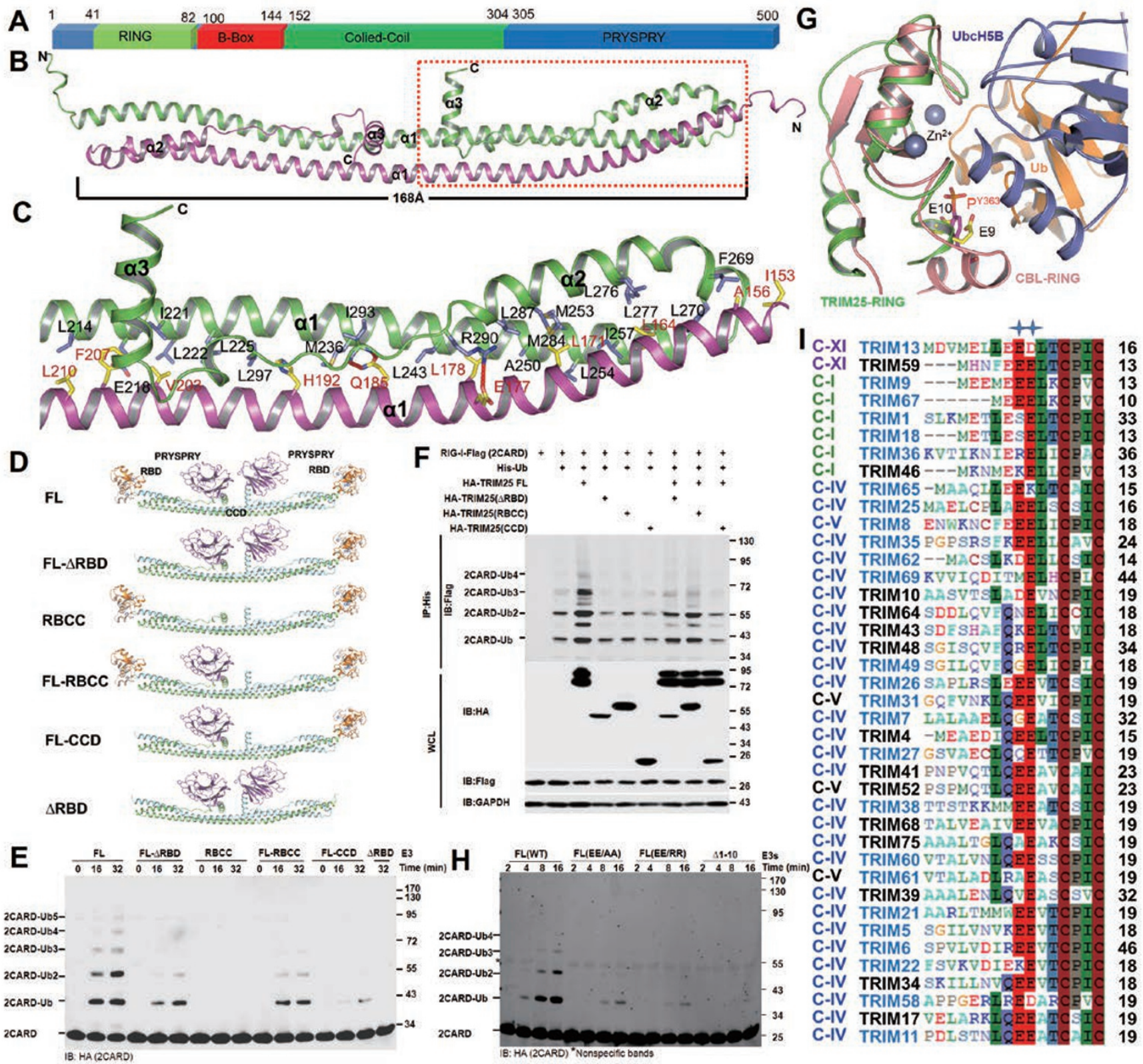

Figure 1 TRIM proteins form an anti-parallel dimer. (A) A schematic view of the TRIM69 domain organization. The residue numbers are indicated above. (B) An overall structure of the TRIM69 CCD dimer. The N- and C-termini are shown. (C) A zoom-in view of the region in the box in Figure 1B, showing the detailed interactions of the conserved residues among TRIM family proteins between the two molecules. Interacting residues of the two molecules are labeled as shown. Red dashed lines represent hydrogen bonds. (D) The models illustrate TRIM25 dimers with different combinations of wild-type protein and various truncations. FL, full length; $\triangle$ RBD, deletion of both RING and B-box domains; RBCC, RING/B-box/CCD; CCD, coiled-coil domain. (E) Both RBDs of the TRIM25 dimer are required for optimal ligase activity in vitro. Ubiquitination assays were performed using the recombinant TRIM25 dimers shown in Figure 1D. (F) Both RBDs of the TRIM25 dimer are required for the ligase activity in HEK293T cells. (G) The conserved Glu residues mimic the pTyr363 of CBL-B. The model of the TRIM25 RING domain is superimposed to the RING domain of CBL-B. The domains of CBL-B-UbcH5B-Ub are shown. The two conserved Glu residues of TRIM25 are shown in yellow and the pTyr363 of CBL-B is shown in magenta. (H) The acidic motif is required for ubiquitination activity in vitro. Both Glu9 and Glu10 residues were mutated to Ala or Arg. (I) TRIM proteins that contain a conserved acidic motif before the RING domain. The subfamilies of the TRIM proteins are indicated. Proteins known to be involved in antiviral defenses are colored in slate. 
TRIM25 forms anti-parallel dimers. The TRIM25 protein forms an elongated shape, similar to TRIM69, and the RBDs (RING and B-box domains) that presumably locate at the ends have different conformations in some particles probably due to their flexibility (Supplementary information, Figure S6D). As shown in the co-immuoprecipitation (co-IP) assays, differently tagged TRIM25 proteins interacted with each other, which is mediated by their CCDs, further supporting the idea that TRIM25 forms dimer (Supplementary information, Figure S6E). Inferring from the structure of TRIM69 CCD, we propose that TRIM25 might also dimerize through its CCD in vivo; each end of the dimer contains one RBD, and the two PRY/SPRY domains might locate at the midpoint as those in TRIM69 (Supplementary information, Figures S1 and S4).

Previous studies suggest that TRIM proteins might form a parallel dimer with the RING domains functioning as one catalytic unit based on spatial approaching [10]. Our structure, however, suggests that the two RING domains in a TRIM dimer are separated by the long CCD, with a distance of about $170 \AA$. To find out how the two spatially separated RING domains work together to catalyze ubiquitination, we constructed a set of TRIM25 dimers with different combinations of wild-type and truncated monomers (Figure 1D and Supplementary information, Figure S7). Consistent with the previous report [7], FL TRIM25 homodimer ubiquitinated the CARDs of RIG-I. The FL- $\triangle$ RBD and FL-RBCC complexes had impaired activity. FL-CCD dimer had very low activity. RBCC and $\triangle \mathrm{RBD}$ proteins had no detectable activity (Figure 1E). Furthermore, co-IP results indicate that the PRY/SPRY domain of TRIM25 is required for substrate recruitment (Supplementary information, Figure S8). Consistently, the FL-RBCC dimer exhibited a lower activity than the FL homodimer, whereas the RBCC dimer had no detectable activity. The in vivo ubiquitination results were consistent with the in vitro observations (Figure 1F). Interestingly, the SCFCdc4 ubiquitin ligase complex also forms a superhelical homotypic dimer through Cdc4 proteins, and similarly each protein at both ends with a distance of about $160 \AA$ is required for unbiquitination [11]. It might be a common feature of E3 ligases that the two reactive centers at each end of an antiparallel dimer work co-operatively.

Comparison of the ubiquitination activities of TRIM25 dimers of various combinations has three implications. First, a single RBD at one end of a dimer could function individually. Second, the RBD at one side could ubiquitinate the substrate bound to both PRY/SPRY domains, but ubiquitinates the substrate bound to the proximal PRY/SPRY domain more efficiently. Third, each PRY/
SPRY domain could bind substrate individually. The time course assay showed that TRIM25 dimers with two RBDs were much more efficient in catalyzing ubiquitination as compared to the dimers with a single RBD (Supplementary information, Figure S9A and S9B). The activity of the FL homodimer was not inhibited by adding $\triangle \mathrm{RBD}$ proteins indicating that this dimer did not dissociate or exchange with free monomers (Supplementary information, Figure S9C-S9F). Together, these results suggest that two RBDs in a FL-TRIM25 dimer could work cooperatively to ubiquitinate CARDs (2CARD), consistent with previous studies suggesting an allosteric mechanism of ubiquitin transfer by RING domaincontaining ubiquitin E3 ligase proteins [12].

To understand how a single RBD could exert ubiquitin ligase activity, we further truncated TRIM25 RING, $\mathrm{B} 1, \mathrm{~B} 2$ or both $\mathrm{B}$ domains. The in vivo results showed that both $\mathrm{B} 1$ and $\mathrm{B} 2$ were required for efficient substrate ubiquitination (Supplementary information, Figure S10). A recent study of CBL-B revealed that a phosphorylated tyrosine-containing element enhances its catalytic efficiency by about 200-fold [13]. A structure model of TRIM25 RING domain was built based on the structure of RNF168 (PDB: 3L11), which shares 43\% sequence identity with TRIM25. Interestingly, two highly conserved glutamates (Glu9 and Glu10) in TRIM25 were located in a site equivalent to the phospho-tyrosine in CBL-B (Figure 1G), raising the possibility that the two Glu residues might play a similar role as the phosphotyrosine of CBL-B in promoting the ubiquitination activity of TRIM25. Consistent with the structure-based prediction, in vitro and in vivo ubiquitination assays suggest that these acidic residues are required for the ubiquitin ligase activity of TRIM25 (Figure 1H and Supplementary information, Figure S11). Sequence alignment indicates that about 40 TRIMs have an acidic motif containing one or two conserved Glu residues immediately ahead of the RING domain (Figure 1I). Among them, 26 are involved in the innate immune responses (Figure 1I) and contain the PRY/SPRY domain [9, 14]. Whether this conserved acidic motif is required for catalytic activity of other TRIMs needs further investigation.

In conclusion, our results show that TRIM proteins form anti-parallel dimers with two spatially separated RING domains acting cooperatively to ubiquitinate substrates. Our study also identified a conserved acidic motif of TRIM proteins that might regulate the enzymatic activity. The rigidly conserved combination, pattern, and order of the tripartite domains among TRIM proteins suggest that the model described here is likely applicable to other TRIM members. It would be interesting to test in future studies whether a similar mechanism is shared by 
other members of this fascinating family of E3 ligases, and to explore the differences that enable TRIM proteins to perform diverse functions. Of note is that during preparation of our manuscript, a similar conclusion was made based on the crystal structure of the anti-parallel dimer of TRIM25-CCD [15].

\section{Acknowledgments}

We thank Shilong Fan and the staff at the SSRF BL17U beamline for their assistance in data collection. We thank Jijie Chai and Yigong Shi from Tsinghua University for critical reading of the manuscript. This work was supported by the National Basic Research Program of China (973 Program; 2011CB910502, 2012CB911101 and 2011CB944302), the National Natural Science Foundation of China (31030020 and 31170679) and the State Key Laboratory Special Fund (2060204).

Yang $\mathrm{Li}^{1,2,{ }^{*}}$, Han $\mathrm{Wu}^{1,2, *}$, Wei $\mathrm{Wu}^{3, *}$, Wei Zhuo ${ }^{1,2, *}$, Weixiao Liu ${ }^{4}$, Yixiao Zhang ${ }^{1}$, Minzhang Cheng ${ }^{1,2}$, Ye-Guang Chen ${ }^{1,2}$, Ning Gao ${ }^{1}$, Hongtao $\mathrm{Yu}^{5}$, Linfang $\mathrm{Wang}^{3}$, Wei Li ${ }^{4}$, Maojun Yang ${ }^{1,2}$

${ }^{I}$ MOE Key Laboratory of Protein Sciences, Tsinghua-Peking Center for Life Sciences, School of Life Sciences, Tsinghua University, Beijing 100084, China; ${ }^{2}$ Department of Pharmacology and Pharmaceutical Sciences, School of Medicine, Tsinghua University, Beijing 100084, China; ${ }^{3}$ State Key Laboratory of Medical Molecular Biology, Institute of Basic Medical Sciences, Chinese Academy of Medical Sciences, Peking Union Medical College, Tsinghua University, Beijing 100005, China; ${ }^{4}$ State Key Laboratory of Reproductive Biology, Institute of Zoology, Chinese Academy of Sciences, Beijing 100101, China; ${ }^{5}$ Howard Hughes Medical Institute, Department of Pharmacology, University of Texas Southwestern Medical Center, Dallas, TX 75390, USA

*These four authors contributed equally to this work. Correspondence: Maojun Yang ${ }^{\mathrm{a}}$, Wei Li $\mathrm{i}^{\mathrm{b}}$, Linfang Wang ${ }^{\mathrm{c}}$

${ }^{\mathrm{a}}$ Tel: +86-10-6278-9400; Fax: +86-10-6279-2736
E-mail: maojunyang@tsinghua.edu.cn

${ }^{\mathrm{b}}$ Tel/Fax: +86-10-6480-7529

E-mail: leways@ioz.ac.cn

'Tel: +86-10-6224-0803; Fax: +86-10-6224-0529

E-mail: wang.linfang@imicams.ac.cn

\section{References}

1 Hatakeyama S. Nat Rev Cancer 2011; 11:792-804.

2 Doyle JM, Gao J, Wang J, et al. Mol Cell 2010; 39:963-974.

3 Borden KL, Lally JM, Martin SR, et al. Proc Natl Acad Sci USA 1996; 93:1601-1606.

4 Herquel B, Ouararhni K, Khetchoumian K, et al. Proc Natl Acad Sci USA 2011; 108:8212-8217.

5 Reymond A, Meroni G, Fantozzi A, et al. EMBO J 2001; 20:21402151.

6 Chan CH, Li CF, Yang WL, et al. Cell 2012; 149:1098-1111.

7 Gack MU, Shin YC, Joo CH, et al. Nature 2007; 446:916-920.

8 Zhang Z, Bao M, Lu N, et al. Nat immunol 2013; 14:172-178.

9 Versteeg GA, Rajsbaum R, Sanchez-Aparicio MT, et al. Immunity 2013; 38:384-398.

10 Meroni G, Diez-Roux G. BioEssays : news and reviews in molecular, cellular and developmental biology 2005; 27:1147-1157.

11 Tang X, Orlicky S, Lin Z, et al. Cell 2007; 129:1165-1176.

12 Streich FC Jr, Ronchi VP, Connick JP, et al. J Biol Chem 2013; 288:8209-8221.

13 Dou H, Buetow L, Sibbet GJ, et al. Nat Struct Mol Biol 2013; 20:982986

14 Kawai T, Akira S. EMBO Mol Med 2011; 3:513-527.

15 Sanchez JG, Okreglicka K, Chandrasekaran V, et al. Proc Natl Acad Sci USA 2014; 111:2494-2499.

(Supplementary information is linked to the online version of the paper on the Cell Research website.)

(i) (s) (-) This work is licensed under the Creative Commons Attribution-NonCommercial-No Derivative Works 3.0 Unported License. To view a copy of this license, visit http:// creativecommons.org/licenses/by-nc-nd/3.0 